\title{
Book reviews : A la recherche de la qualité de la ville durable: Ies ressources du «projet par la recherche»
}

\author{
Jean-Bernard Racine \\ Université de Lausanne Grey 16, 1004 Lausanne, Switzerland \\ Correspondence: Jean-Bernard Racine (jean-bernard.racine@unil.ch) \\ Published: 13 May 2019
}

Bailly, E. (Sous la direction de) : Oser la ville sensible, Cosmografia, Nantes, 196 pp., Préface de Paquot, T., ISBN : 9782955004876, EUR 26, 2018

Bailly, E. et Marchand, D. (Sous la direction de) : Penser la qualité : La ville résiliente et sensible, Editions Mardaga, Bruxelles, 245 pp., ISBN : 9782804706609, EUR 28, 2019

«La pensée est une aventure. La seule peut-être aujourd'hui à découvrir des espaces nouveaux ». Ainsi s'exprimait le philosophe Yves Charles Zarka en introduisant une collection d'articles des principaux philosophes européens de notre temps, réunis dans un remarquable hors-série paru aux Presses universitaires de France sous le titre Voyages inédits dans la pensée contemporaine à l'occasion du dixième anniversaire de la revue Cités (2010). L'expression est revenue en mémoire du soussigné à la lecture inattendue pour lui des deux ouvrages animés et co-dirigés par urbaniste Emeline Bailly, chercheuse sur la qualité des villes au Centre scientifique et technique du bâtiment (CSTB) et chercheuse associée au Lab'Urba de l'Ecole d'urbanisme de Paris, conduisant des recherches sur la conception urbaine durable et les conceptions de paysage, d'urbanité et d'accueillance des lieux.

Comme le rappelait récemment l'Institut de géographie et durabilité de l'Université de Lausanne en invitant praticiens, chercheurs et étudiants à une conférence publique donnée par François Leclercq et Bertrand Vignal, architecte urbaniste pour le premier, architecte paysagiste pour le second, présentant des projets de requalification de tissus existants pour penser l'habitabilité des espaces depuis l'échelle métropolitaine jusqu'à celle de l'espace public, la requalification de l'existant est un thème récurrent dans les manières de produire, concevoir et gérer les espaces de vie. Et de préciser : «Qu'il s'agisse de restructuration d'échangeurs au- toroutiers, de pôles-gare, d'entrées de ville pour articuler centralités/qualité des espaces produits et mixité des programmes, ou encore de la création de véritables structures actives d'espaces publics capables de répondre à la transition climatique notamment [...], le motif commun à ces approches de projet a ceci de particulier : le récit du projet urbain alimente la projection de la démarche de transition vers l'habitabilité de nouveaux espaces de vie. De quelles manières les approches paysagères et urbanistiques dans/pour et avec l'existant peuvent-elles être articulées aux approches de qualification des espaces publics ? Comment accompagne-ton la transition ? Pour quel(s) dess(e)ins d'habitabilité ? »

Le hasard nous a permis de prendre connaissance tout à fait indépendamment mais au même moment, des deux ouvrages mentionnés ci-dessus, lesquels répondaient assez exactement à la possibilité d'acquérir une formation en la matière, et ce même s'ils sont signés d'auteurs qui semblent ignorer - dans le premier ouvrage du moins, consacré à la ville sensible -, les contributions spécifiquement géographiques en la matière, à l'exception de celles d'Augustin Berque, contrairement au second qui l'a suivi moins d'un an plus tard autour du thème Penser la qualité et sous-titré La ville résiliente et sensible. Cet ouvrage leur accorde une place, de manière très modeste, quoique fort pertinente. Mais un petit $2 \%$ des renvois bibliographiques, à l'occasion de quelques chapitres uniquement : on retrouvera l'Américain I.F. Thuan bien sûr, l'Anglais M. Pacione, le Canadien E. Relph, le Français Di Méo, à propos de textes réalisés dans le dernier quart du siècle précédent, tout en offrant une place plus importante aux travaux plus récents animés ou réalisés de Suisses romands, A. Bailly, A. Da Cunha et J. F. Staszak. Et pourtant... Quel bel apport pour notre discipline que ces deux ouvrages!

Oser la ville sensible commence par une hypothèse de départ, formulée et reformulée tout au long de l'ouvrage, au 
fil des étapes de ce magnifique essai vécu. «Le paysage sensible, c'est-à-dire tenant compte des sensations, affects, émotions, est une composante clé de la qualité des futurs quartiers aménagés » (p. 107). «Mais comment définir un sentiment de paysage, l'appréhender et le traduire dans les actes de transformation urbaine? » «Un paysage n'existe pas en soi » rappelle le philosophe de l'urbain, Thierry Paquot, dans la belle préface qu'il a offerte à l'ouvrage. En ajoutant que «depuis les premiers usages dans le vocabulaire des peintres occidentaux dès le début du XVIe siècle, le mot «paysage » est associé au regard [...], une suprématie qui se trouve chahutée depuis que l'on commence à admettre qu'un paysage se ressent sensoriellement », mieux, qu'il «participe à l'existence », «l'expérience paysagère s'apparente bien à un cadeau : le don des sensations » (Paquot, pp. 11-14). Et ce, autour de l'idée neuve, venue des architectes et de quelques sociologues, et non pas des géographes, à ceci près que quelques-uns parmi eux, des deux côtés de l'Atlantique, se sont précocement intéressés à la géographie des émotions et en ont eu l'intuition en cherchant à théoriser notre rapport affectif à la ville, il y a quelques années déjà. Quelques articles en témoignent et depuis nombre de recherches et séminaires communs ont fleuri, en France particulièrement. Mais quoique cette thématique ait été reprise ici ou là, elle reste à notre connaissance sans systématisme et sans proposer de terrains d'analyse originaux permettant de suivre pas à pas la recherche évoquée et la manière d'en traduire les résultats de manière à nourrir des politiques opérationnelles. En revanche, avec Oser la ville sensible, les lecteurs entreront de plein pied dans les tours et détours d'une recherche dont ils peuvent suivre les tenants et aboutissants de manière réflexive et critique, comme s'ils y avaient directement participé.

Le géographe lecteur de ces ouvrages découvrira en effet avec un intérêt constant, à la fois admiratif et reconnaissant l'apport des différents auteurs mobilisés pour l'affinage de sa formation d'analyste et de praticien des politiques visant l'habitabilité de nos principaux espaces de vie. On n'imagine pas tout ce qu'il va apprendre - y compris quand les choses ne se concrétisent que difficilement - et qui pour lui devient subitement essentiel. Et ce dès le premier ouvrage, consacré au compte rendu réflexif et critique d'une « rechercheprojet »dont les auteurs donnent au passage une définition fort intéressante, « le projet par la recherche », laquelle à elle seule devrait faire date.

Pour comprendre l'appréhension sensible des paysages sans la simplifier ou l'instrumentaliser, envisager leurs traductions spatiales sans les dénaturer, en tenir compte et les révéler à travers des interventions urbaines et passagères, une belle équipe d'architectes urbanistes a en effet initié en 2015 cette « recherche-projet », Fabrique active de paysages (FACT), portée principalement par le Centre scientifique et technique du bâtiment, soutenu par la Région Île de France, la ville de L'Île-Saint-Denis, la communauté d'agglomération Plaine Commune et la Drac Île de France. L'am- bition explicite étant de dépasser l'idéal abstrait d'une ville sensible, pour esquisser les perspectives d'une intervention « urbaine et paysagère » plus respectueuse des dimensions idéelles et affectives, à même de créer des lieux susceptibles d'être expérimentés et éprouvés, manière d'esquisser in fine « les contours d'une possible conception urbaine que les auteurs ont qualifiée de sensible » (p. 19). Au centre des préoccupations, la qualité du paysage urbain, voire du paysage tout court, tel qu'il est vécu par tout un chacun.

De fait, Oser la ville sensible est un ouvrage organisé de manière à suivre, avec les habitants, dans un langage quotidien et commenté, l'équipe FACT, laquelle pendant trois ans, a révélé «par petites touches », des espaces publics et paysages de ce territoire périphérique du Grand Paris ... par des modes d'interventions urbaines artistiques fondées sur l'expérience sensorielle et émotionnelle des habitants, le réemploi des ressources locales et l'intervention de concepteursartistes de disciplines variées (paysagistes, designers, graphistes, plasticiens, sculpteurs et documentaristes sonores), ayant répondu à un « appel à participer » et offrant, l'un un projet de module sur les berges, l'autre un ponton sur l'eau. $\mathrm{Au}$ fil des divers ateliers proposés, successivement présentés et illustrés, où s'est noué le passage («mariage arrangé ») entre recherche et projet, des lieux et micro-paysages ont été révélés - l'ouvrage est à cet égard remarquablement illustré embarquant littéralement le lecteur comme si ce dernier avait pu participer aux ateliers-promenade du départ et à l'analyse des lieux et des appréhensions paysagères, voire à la discussion des propositions d'aménagement. Des propositions d'artistes, toutes modestes, apparemment localement pertinentes dans leur originalité même de lieux ménagés, mis en lumière, selon des protocoles scientifiques et des prototypes d'installations urbaines singuliers...

A cet égard, ce compte rendu d'une recherche que d'aucuns assimileront peut-être à un modèle de recherche-action, mais le dépassant largement par la place accordée à la réflexion théorique et critique qui l'accompagne en amont et en aval, se présente comme un modèle de nouveau type d'ouvrage, expression d'une véritable aventure de la pensée à la recherche d'une définition de la «qualité urbaine » en considérant et illustrant de manière totalement originale d'autres dimensions de la ville, celles immatérielles qui fondent ce « sensible » cher à Pierre Sansot, en interrogeant le sens de ce qui fait l'essence des lieux et plus encore de leurs paysages, compte tenu du fait que les paysages participent - au sens le plus fort - à l'existence, à notre existence. Et ce, singulièrement dans la mesure où, nous dit Emeline Bailly dans sa propre introduction (p. 17) : « ils sont au cœur du rapport sensible et affectif aux espaces urbains, dans la mesure où ils questionnent les sentis et ressentis associés aux lieux, les subjectivités partagées qu'ils peuvent susciter et les potentiels descripteurs urbains du monde sensible ». Reste à savoir si dans le cadre de cette interrogation, nous assistons à un renouveau de la conception urbaine. 
Et c'est bien ce que s'efforce de démontrer, avec plus d'amplitude et cette fois sans ignorer certains travaux proprement géographiques, l'ouvrage suivant co-dirigé et co-signé encore par Emeline Bailly accompagnée cette fois par Dorothée Marchand, docteur en psychologie sociale et environnementale, chercheuse au Centre scientifique et technique du bâtiment (CSTB), associées à une dizaine d'autres auteurs ou co-auteurs des différents chapitres, - un co-auteur géographe tout de même - quatre au sein d'une première partie intitulée Approche critique et conceptuelle de la qualité (pp. 22115), incluant des chercheurs issus de domaines scientifiques différents (urbanisme, psychologie environnementale, philosophie, architecture et même géographie cette fois); quatre encore pour la deuxième partie, Les dimensions clés de la qualité urbaine (pp. 116-178), laquelle évoque successivement - une fois rappelée la relativité de la notion de qualité selon les cultures, les époques les lieux -, la place de la nature, comme dimension des environnements et moyen de ressourcement pour les habitants, la marchabilité, la qualité technique enfin, en relation avec la qualité innovante.

Une troisième partie est alors constituée, sous le titre significativement programmatique $\hat{E}$ tre en ville, être urbain, par des entretiens avec psychologues, ingénieurs spécialisés dans le bâtiment, philosophes de l'urbain (on retrouvera Thierry Paquot, inévitable en la matière), artistes et urbanistes paysagistes voire « végétalistes ». Superbe aréopage pour «penser la qualité » à propos de la ville, cette fois doublement qualifiée - disons, en amont et en aval de ce que ses habitants peuvent en attendre - de « résiliente » et de « sensible », l'ensemble organisé autour de l'hypothèse initiale voulant que « la notion de qualité masque un impensé urbain, celui de l'appréciation qualitative des lieux ». D'où l'idée force soustendant tout l'ouvrage et imposant son importance : le fait que « conceptualiser la qualité offrirait alors une opportunité de renouvellement de la pensée urbaine et, par extension, nos perspectives d'expériences d'être urbains dans le monde » (p. 12). L'essentiel est dit. C'est du moins la forte impression que cette lecture a pu produire sur le soussigné, géographe certes retraité des responsabilités d'enseignement et de recherche, mais non de la participation à la réflexion sur le « repositionnement du lieu social au cœur de la société urbaine », « pour répondre aux défis du développement durable » comme le dit justement (p. 10) Alain Maugard, ancien directeur de la Construction au Ministère français du logement et du Centre scientifique et technique du bâtiment (CSTB). D'une phrase, il indique tout l'intérêt de l'ouvrage, lequel, accessoirement, fera la part belle aux pratiques participatives. A bien des égards, il est l'ouvrage programmatique que nous attendions, qu'il s'agisse de nourrir un enseignement, ou de servir de fondement à l'orientation de nos recherches.

Reste à s'interroger sur un élément du sous-titre, le concept de « résilience », proposé à côté de celui de «sensible », mais qui n'est évoqué que quatre fois, au début, au milieu et deux fois à la fin - mais à peine - sans jamais être explicité. C'est peu pour un sous-titre, à l'heure où se multiplient partout - c'est la mode - et même autour de Lausanne, des deux côtés de l'Atlantique, les références à ces villes qui se veulent et se disent déjà « résilientes » : sauf à se dire que dans ce remarquable ouvrage, il nous est proposé indirectement - sans doute l'apport de la co-directrice, docteure en psychologie sociale et environnementale - comme un fil directeur de l'ouvrage, les auteurs estimant que la qualité est un facteur essentiel de la résilience. Cette qualité urbaine qu'Emeline Bailly définit si bien (p. 235) comme étant « aussi liée à la restauration qui concourt à redonner une identité et à ré-enraciner dans la ville » ? 